\title{
4EBP1/2 are active under standard cell culture conditions to regulate the translation of specific mRNAs
}

\author{
Khawla Alasad (10), Kai Voeltzke², Liron Levin ${ }^{3}$, Guido Reifenberger ${ }^{2,4}$, Gabriel Leprivier ${ }^{2}$ and Barak Rotblat (10 ${ }^{1,3}$
}

The mammalian target of rapamycin (mTOR) kinase is a nutrient sensor coordinating cellular anabolic and catabolic processes ${ }^{1}$. During favourable metabolic conditions, mTOR promotes protein synthesis by phosphorylating its substrates, including eIF4E binding proteins 1-3 (4EBP1-3). Upon conditions where mTOR is inactive, the hypo-phosphorylated and active 4EBPs bind to eukaryotic initiation factor $4 E$ (eIF4E), competing with the recruitment of eIF4G thus disrupting the formation of the eIF4F complex, in turn leading to inhibition of cap-dependent translation initiation ${ }^{2}$.

It is not known whether 4EBPs regulate mRNA translation in optimal growth conditions, in which mTOR is active and 4EBPs thus phosphorylated and presumed to be inactive. This question is particularly relevant in pathological and physiological conditions where the expression of 4EBPs are up- or down-regulated while mTOR is active.

To assess the activity of 4EBP $1 / 2$ under basal cell culture conditions, we used lysates of 4EBP1/2 knockdown (KD) and control scramble shRNA (shSCR) HEK293 cells $^{3}$ to pull down eIF4E and its interacting proteins using $\mathrm{m}^{7}$ GTP-agarose beads (Fig. 1a). We found more eIF4G bound to eIF4E in KD lysates as compared to shSCR cell lysates (Fig. 1a). This finding was confirmed using 4EBP1/2 WT and double KO (DKO) $\mathrm{p}^{-1-} 3^{-1} \mathrm{MEFs}^{3}$ (Fig. 1a), suggesting that an active cellular fraction of 4EBPs is detectable in optimal cell culture conditions, even in the presence of active mTOR.

\footnotetext{
Correspondence: Barak Rotblat (rotblat@bgu.ac.il)

'Department of Life Sciences, Ben-Gurion University of the Negev, Beer Sheva, Israel

${ }^{2}$ Institute of Neuropathology, Medical Faculty, University Hospital Düsseldorf, Düsseldorf, Germany
}

Full list of author information is available at the end of the article
However, we did not find a statistically significant effect of the active 4EBPs fraction on overall protein synthesis using AHA labelling ${ }^{4}$ under basal conditions, although there was a trend towards increased protein synthesis in DKO cells (Fig. S1a). Nevertheless, using a bicistronic reporter vector in which Renilla luciferase (Rluc) is translated in a cap-dependent manner, while Firefly luciferase (Fluc) is translated in a cap-independent manner (Fig. 1b) $)^{5}$, we found that KD cells exhibited a significantly higher Rluc/Fluc ratio as compared to controls (Fig. 1b). These data suggest that while 4EBP1/2 restrict capdependent translation in normal cell culture conditions, this has minimal impact on overall protein synthesis, pointing to a selective regulation of mRNA translation.

We then identified transcripts whose translation is selectively influenced by $4 \mathrm{EBP} 1 / 2$ under basal conditions, by performing polysome profiling using a non-linear sucrose gradient (the Larsson protocol) ${ }^{6}$ (Fig. 1c). Total and polysomal mRNA, obtained from KD and shSCR cells, were identified and quantified by RNAseq. Analysis of total mRNA expression showed that only 26 genes were differentially expressed between KD and shSCR cells (Fig. S1b and Supplementary Table 1). We calculated the translation efficiency (TE) of each mRNA as the ratio between polysomal and total mRNA levels in KD and shSCR samples (Fig. S1c) and found 516 transcripts with lower TE in KD cells (cluster \#1) and 569 transcripts whose translation was increased in KD cells (cluster \#2) (Supplementary Table 1). KEGG analysis of transcripts whose TE was affected by $4 \mathrm{EBP} 1 / 2$ identified pathways previously linked to $4 \mathrm{EBP} 1 / 2$ functions including ribosomes, oxidative phosphorylation, metabolic pathways and neurodegeneration (Fig. S1d) ${ }^{3,7,8}$. Overall, these data show that 4EBP1/2 selectively affect the translatome in normal cell culture conditions. 


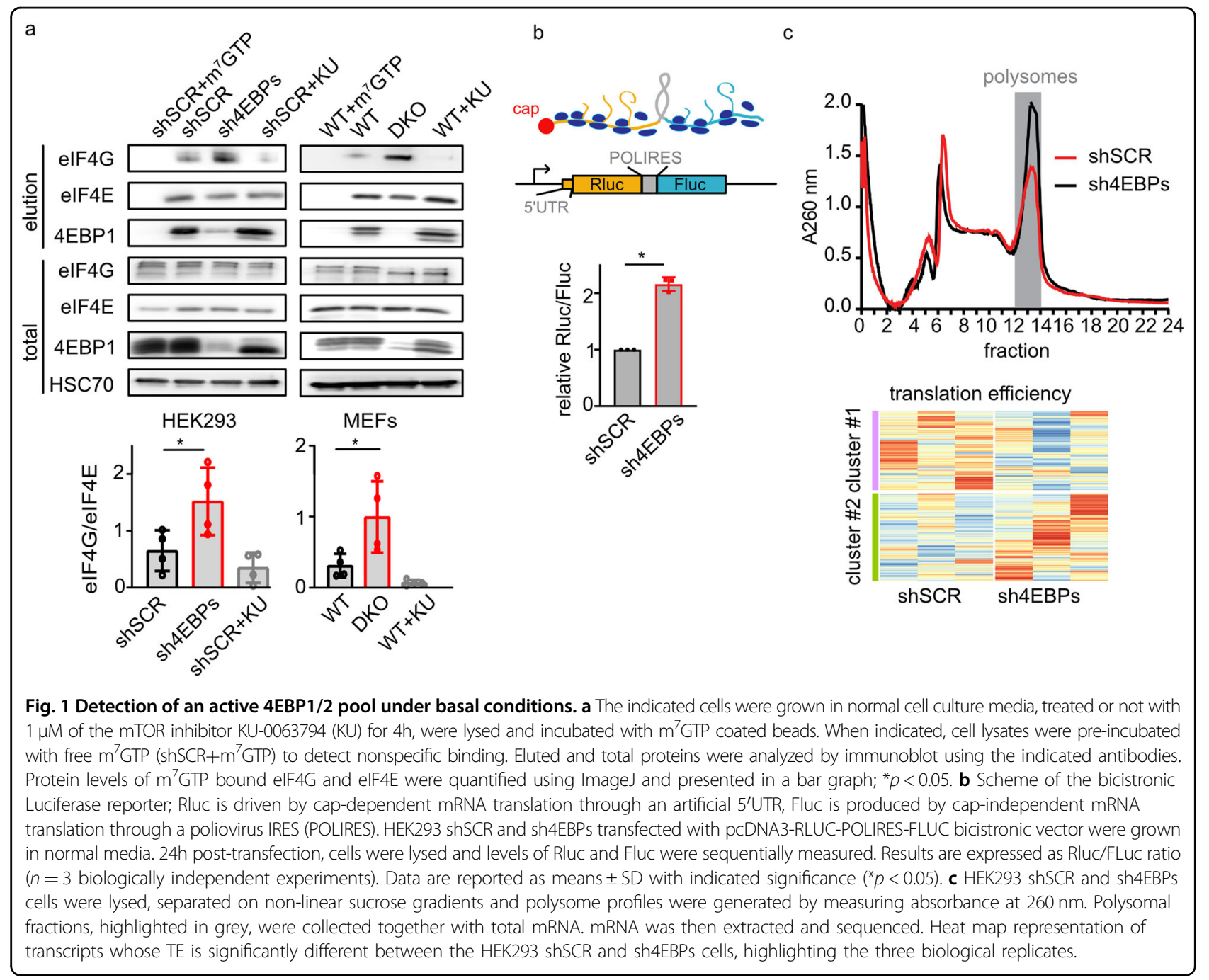

\section{Acknowledgements}

This research was supported by the Israel Science Foundation (grant No. 1436/ 19), by the Israeli Cancer Association (grant \#20180012) and the NIBN. G.L. is supported by the Deutsche Forschungsgemeinschaft (LE 3751/2-1), the German Cancer Aid (70112624) and the Elterninitiative Kinderkrebsklinik.

\section{Author details}

'Department of Life Sciences, Ben-Gurion University of the Negev, Beer Sheva, Israel. ${ }^{2}$ Institute of Neuropathology, Medical Faculty, University Hospital Düsseldorf, Düsseldorf, Germany. ${ }^{3}$ The National Institute for Biotechnology in the Negev, Beer Sheva, Israel. ${ }^{4}$ German Cancer Consortium (DKTK), partner site Essen/Düsseldorf, Düsseldorf, Germany

\section{Conflict of interest}

The authors declare that they have no conflict of interest.

\section{Publisher's note}

Springer Nature remains neutral with regard to jurisdictional claims in published maps and institutional affiliations.

Supplementary Information accompanies this paper at (https://doi.org/ 10.1038/s41419-020-03182-6).
Received: 27 July 2020 Revised: 9 October 2020 Accepted: 13 October 2020 Published online: 11 November 2020

\section{References}

1. Kim, J. \& Guan, K.L. mTOR as a central hub of nutrient signalling and cell growth. Nat. Cell Biol. 21, 63-71 (2019).

2. Haghighat, A., Mader, S., Pause, A. \& Sonenberg, N. Repression of capdependent translation by $4 \mathrm{E}$-binding protein 1: competition with p220 for binding to eukaryotic initiation factor-4E. EMBO J. 14, 5701-5709 (1995).

3. Dowling, R. R. J. O. et al. mTORC1-mediated cell proliferation, but not cell growth, controlled by the 4E-BPs. Science 328, 1172-1177 (2010).

4. Marciano, R., Leprivier, G. \& Rotblat, B. Puromycin labeling does not allow protein synthesis to be measured in energy-starved cells correspondence. Cell Death Dis. https://doi.org/10.1038/s41419-017-0056-x (2018).

5. Tsukumo, Y., Sonenberg, N. \& Alain, T. Transcriptional induction of 4E-BP3 prolongs translation repression. Cell Cycle 0, 1-2 (2016).

6. Liang, S. et al. Polysome-profiling in small tissue samples. Nucleic Acids Res $\mathbf{4 6}$ 1-13 (2017).

7. Thoreen, C. C. et al. A unifying model for mTORC1-mediated regulation of mRNA translation. Nature 485, 109-113 (2012).

8. Hsieh, A. C. et al. The translational landscape of mTOR signalling steers cancer initiation and metastasis. Nature 485, 55-61 (2012). 